\title{
Interfacial Interactions of Phosphatidylglycerol with Oligonucleotide DNA Revealed by Molecular Dynamics Method
}

\author{
Milyausha Y. Ibragimova 1,2 (D), Rustam Kh. Aupov 1(D), Grigorii V. Andrianov 1(D), \\ Renad I. Zhdanov 1,2 (D), Sergei Yu. Zaitsev 3,* (D) \\ 1 Kazan (Volga region) Federal University, Institute of Fundamental Medicine and Biology, 18, Kremlevskaya St., Kazan \\ 420008, Russian Federation; milyausha_ibrahim@mail.ru (M.Y.I.); \\ 2 Moscow State Pedagogical University, Russian Institute for Advanced Study RIAS, 1, Malaya Pirogovskaya St., Moscow \\ 119991, Russian Federation milyausha_ibrahim@mail.ru (M.Y.I.); \\ 3 Federal Research Center for Animal Husbandry named after Academy Member L.K. Ernst, Dubrovitsy 60, Podolsk \\ Municipal District, Moscow Region, 142132 Russian Federation, 142132 Russian Federation; s.y.zaitsev@mail.ru \\ (S.Yu.Z.); \\ * Correspondence: s.y.zaitsev@mail.ru (S.Yu.Z.);
}

Scopus Author ID 7103012267

Received: 31.05.2021; Revised: 5.07.2021; Accepted: 10.07.2021; Published: 8.08.2021

\begin{abstract}
An interaction of DNA with lipids is of great interest because of their functions. As fatty acids and lipids can specifically bind to nucleic acids forming a code sequence of the genomic DNA, it is important to study the interaction of the oligonucleotide DNA $(\mathrm{dA})_{20^{\circ}}(\mathrm{dT})_{20}$ with phosphatidylglycerol by the molecular dynamics method. Molecular docking has shown that these components form a stable complex with $5.8 \mathrm{kcal} / \mathrm{mole}$ binding energy, wherein the lipid is located in the DNA minor groove. This configuration marks 354 atom groups separated by a distance less than $3.4 \AA$. The van der Waals and hydrophobic interactions play the leading part in the DNA-phospholipid complex stabilization along with hydrogen bonds.
\end{abstract}

Keywords: interfacial interactions; interatomic distances; phosphatidylglycerol; oligonucleotides; DNA-lipid complexes; docking; molecular dynamics

(C) 2021 by the authors. This article is an open-access article distributed under the terms and conditions of the Creative Commons Attribution (CC BY) license (https://creativecommons.org/licenses/by/4.0/).

\section{Introduction}

The interfacial interactions of DNA with lipids are of great interest in connection with the functions they perform in cells: from the structural components of cell membranes and cellular organoids to signaling molecules in the processes of transcription, replication, and repair [1, 2]. Obviously, lipidomics is actively developing in large-scale projects: "Lipid Metabolites and Pathways Strategy (LIPID MAPS)", "COBRE in lipidomics and pathobiology", "Kansas Lipidomics Research Center" at the University of Kansas (USA) etc. However, these projects do not manifest enough attention to studying cell nucleus phospholipids, particularly DNA-bound lipids [3]. At the same time, there are fractions of phospholipids tightly bound to genomic DNA [4], which bind to DNA sequence specifically [5]. It is assumed that such lipids can form a new information level in genomic DNA. DNAmembrane and DNA-lipid interactions and complexes are realized in DNA-membrane contacts, in eukaryotic chromatin located at the inner nuclear membrane around nuclear pores, during signal transmission, as well as in mitochondria [6, 7]. We performed computer 
calculations as part of the molecular mechanics' approach to assess the stability of DNA complexes with linoleic and other C18 fatty acids in minor or major grooves [4,7].

The choice of phosphatidylglycerol (PG) as a model lipid molecule is connected with its presence in the majority of prokaryotic cells as one of the simplest natural phospholipids (consisting of glycerol, fatty acids, and phosphate "building blocks" [8-12]). Phosphatidylglycerol is an important lipid not only in various bacteria (as one of the major components of the membranes), but also in plants (as a valuable part of the photosynthesis thylakoids) and animals (as pulmonary surfactant spreading over the lung cellular surface area, etc.) [10]. Two PG molecules by some biosynthetic processes can form cardiolipin (CL), the constituent molecules of cell walls and membranes of some bacteria and the mitochondria [11]. For example, the contents of PG/CL (\% in the membranes of some bacteria) are the following: 46.1/38.8 (Micrococcus lysodeikticus), 58.8/31.0 (M. tetragenus), 74.5/25.5(M. roseus), 64.6/34.8 (M. roseus R27), 88.1/9.6 (M. varlas), 62.7/31.0 (Micrococcus sp.), 69.5/27.4 (M. conglomerates), 52.5/10.0 (M. caseolyticus), 61.7/2.0 (M. rhodochrous), 55.6/36.5 (Sarcina flava), 89.6/1.1 (S. lutea), 31.4/14.5 (S. ventriculi), 32.2/3.0 (S. maxima), 72.1/27.9 (S. ureae), 77.8/3.6 (Bacillus subtilis) [9-14]. Thus, PG is a very important lipid for various membrane modeling systems and methods [8-14], as well as the part of the formulations for inactivation of SARS-CoV-2 ("a potential treatment for COVID-19") and other "enveloped viruses" [1519].

In the recent works [20-26] "a high-throughput molecular dynamics (MD) simulation" approaches for BAS complexes were proposed, and "the output from AutoDock Vina" was used to improve docking results [20-26].

From these data and according to experimental computer results, using the molecular docking method $[4,5,7]$, it follows that DNA complexes with fatty acids in the minor groove are most stable. The same result was obtained in the study of molecular dynamics and free energy of DNA binding with linoleic acid [7]. The creation of models of DNA complexes with fatty acids, especially phospholipids, is an important achievement ("at the junction" of colloidal and supramolecular chemistry) both in fundamental and applied aspects. The mathematical description of such complexes is a fundamental achievement in the field of "surface sciences". The obtained data can be considered as a reference for comparison with experimental values that facilitate their practical use in biomedical fields.

The aim of this work is to study the interaction of phosphatidylglycerol with DNA oligonucleotide $(\mathrm{A})_{20} \bullet(\mathrm{T})_{20}$ by docking and molecular dynamics methods.

\section{Materials and Methods}

\subsection{The measurements of the structure of ligands and complexes.}

The structural parameters of phosphatidylglycerol (PG) with two linoleic acid residues (containing two double bonds each residue) were obtained from the HIC-Up database [27], and the structures were constructed using the Avogadro molecule reader. The structures of doublechain oligonucleotides consisting of $20 \mathrm{~A}-\mathrm{T}$ pairs $(\mathrm{dA})_{20} \bullet(\mathrm{dT})_{20}$ were generated using the NAB utility programs from the AmberTools software package ("AMBER 11, University of California, San Francisco, 2010" [28-32]). The atomic coordinates of the complexes are given in accordance with the generally accepted nomenclature [28].

To obtain the initial structures of the complexes, the PG was placed in a DNA minor groove (in their crystallographic configurations) using the VMD program. The complex was 
dissolved in a rectangular periodicity cell using the TIP3P model of water molecules so that the distance between periodic images of the complex was at least $15 \AA$. To ensure the electro neutrality of the system, the necessary amount of sodium ions was added. For the resulting structure, energy was minimized using the conjugate gradient method in the NAMD program [29]. When generating the DNA structure, the AMBER99 program was used [30], and the GAFF ("General Amber Force Field" [28-32]) program with AM1-BCC charges [4, 7, 31-32] was used to characterize the ligands.

\subsection{Molecular docking.}

To identify the energetically favorable position of phosphatidylglycerol in the DNA grooves and obtain the complexes' initial structures, molecular docking of PG in DNA (in their crystallographic configurations) was carried out using AutoDock with the vina.exe application $[4,33]$.

\subsection{Molecular dynamics.}

The molecular dynamics trajectory was calculated using the NAMD software package [29]. The bond lengths were fixed using the SHAKE algorithm, which allowed using a trajectory calculation time step 2 fs. All trajectories were calculated for a temperature of 300 $\mathrm{K}$. Before calculating the free binding energy and collecting parameters, and the system was brought to equilibrium for $200 \mathrm{ps}$. This time was chosen in our previous experiments [4, 7] and remained the same in all our works to compare the data obtained correctly. Moreover, the proposed time of 200 ps exceeded the equilibrium times, commonly used in most of the modeling experiments of molecular dynamics (see http://ambermd.org/tutorials/basic/tutorial1/section5.htm as an example). The resulting trajectories were analyzed using the VMD software package, as well as using additional scripts written in Python $[34,35]$. When analyzing non-covalent contacts between fatty acid and oligonucleotide atoms, we considered the atoms, the distance between which did not exceed $3.4 \AA$ (a value of $3.4 \AA$ corresponds to the maximum of the van der Waals attraction between carbon atoms) [7].

\section{Results and Discussion}

\subsection{Results.}

Previous work on the DNA interaction with linoleic acid [7] confirmed only the fact of complex formation. We showed the binding of oleic acid along a minor groove of the DNA both by spectroscopy methods and computer simulation. For phosphatidylethanolamine, the interaction of phospholipids with the DNA was previously studied by molecular dynamics and docking methods. In our computer experiments, the result of PG molecule docking with a DNA oligomer $(\mathrm{dA})_{20} \bullet(\mathrm{dT})_{20}$ (see the methods section) was the following: fatty acids of PG were paced into the DNA minor groove, at that the binding energy is $5.8 \mathrm{kcal} / \mathrm{mol}$. Our previous work [4] determined the binding energy for the DNA oligomer complex with phosphatidylethanolamine, which was $6.3 \mathrm{kcal} / \mathrm{mol}$ [4].

The structure of the B-form of a DNA oligomer with PG molecule in the DNA minor groove (obtained as a result of docking) is presented in Figure 1. We calculated the trajectories of atoms in the PG complex (with two linoleic acid residues) with a DNA oligomer by 
molecular dynamics methods as previously described in the works for DNA complexes with linoleic acid $[4,7]$. The results of a change in the "root-mean-square deviation (RMSD) " [4] of the structure coordinates over time are presented in Figure 2. Dynamics results showed good stability of the DNA-lipids complex. At the beginning of the dynamics, an insignificant change in the vibration of the molecules was observed. In the middle of the dynamics, the system fluctuated within insignificant limits $( \pm 1.5 \AA$ ) $)$. At the end of the dynamics, the system fluctuated within 3 angstroms. No sharp changes were noted in the molecular vibrations; the only exception is a change in the lipid RMSD at the end of the dynamics by about $3 \AA$.

We have analyzed the first and the last saved molecular dynamics steps by the number of pairs of atoms, the distance between which is less than $3.4 \AA$. At the first saved step of the dynamics, with the distance between atoms of less than $3.4 \AA$, we detected a total of 343 pairs of atoms. It is important to highlight that only 90 of such pairs are of interest because only these atoms can be pronounced interactions due to the specific distances between them. These are the pairs of O-H (60), N-H (15), O-N (1), N-C (1), O-C (5), O-O (5) atoms, as well as the pairs $\mathrm{P}-\mathrm{O}$ (1) and $\mathrm{P}-\mathrm{H}$ (2). If we compare them with the number of pairs of atoms during docking, there are 11 pairs fewer.

At the last saved step of the dynamics, with the distance between atoms of less than 3.4 $\AA$, we detected a total of 187 pairs of atoms. But only 42 of such pairs are of interest because only these atoms are capable of pronounced interactions due to the specific distances between them. These are the pairs of $\mathrm{O}-\mathrm{H}(34), \mathrm{N}-\mathrm{H}(1), \mathrm{O}-\mathrm{C}(4), \mathrm{O}-\mathrm{O}(1)$ atoms, as well as the pairs P$\mathrm{H}(2)$.

We also found the number of "interatomic" interactions by additional calculation, i.e., the number of pairs of atoms, the distance between which during the course of dynamics was less than $3.4 \AA$. The largest number of such pairs of atoms (namely, 354) turned out to be at the $39^{\text {th }}$ saved step of dynamics.

The minimum number of atomic pairs (equal to 151) is noted at the end of dynamics, whereas about 434 atomic pairs are noted at the initial dynamics steps. At the same time, $8.79 \%$ of the dynamics steps had up to 200 pairs of atoms (the distance between which was less than $3.4 \AA$ ); $46.75 \%$ had from 200 to 250 pairs; $16.08 \%$ - from 250 to 300 pairs; $19.28 \%$ - from 300 to 350 pairs; $9.09 \%$ - from 350 and more pairs. If we consider the number of atomic pairs in the dynamics time, the greater number of atomic pairs falls on the first half of the dynamics. After a sharp change in the lipid RMSD, there are on average 80 atomic pairs less in the second half of the dynamics.

To analyze the mutual orientation of the molecules in the DNA - PG complex, we selected six pairs of DNA and PG atoms. The coordinates of the O4'(166)-H4(1331) pair fluctuate strongly and, apparently, it could appear in this sample by chance (since the necessary distance between them, less than $3.4 \AA$, is observed several times during the dynamics' process) that follows from the data presented at Figure 3. The oxygen atom (LIG41:O2) of the lipid is located between two DNA atoms: the hydrogen atom (DT31:H1) (the average distance all along the entire dynamics is $2.54 \AA$ ) ) and the hydrogen atom (DT33:H4) (2.77 $\AA$ ) (Figure 4). Moreover, the oxygen atom of the lipid is located closer to one of the hydrogen atoms by 0.23 Á (Figure 4). The hydrogen atom of the lipid (LIG 41:H49) is located between two DNA atoms: the oxygen atom (DA10:04) and the nitrogen atom (DA9:N3) with the distance between oxygen $-2.84 \AA$, nitrogen $-2.79 \AA$ (the contact with nitrogen is closer by $0.05 \AA$ ). 


\subsection{Discussion.}

The results of studying the molecular dynamics of the PG-DNA complex indicate that this complex is a stable structure. Moreover, the dynamics of the PG-DNA complex are characterized by low conformational mobility of the PG ligand in DNA. Apparently, the main explanation for this is that the phospholipid is bound to the DNA molecule by two residues of fatty acids.

The PG binding energy in the "minor groove" [4] of the oligodeoxyribonucleotide (A) $20^{\bullet}(\mathrm{T})_{20}$ is $5.8 \mathrm{kcal} / \mathrm{mol}$. The structure of the DNA complex with the PG molecule in the minor groove (Figure 1) has been obtained for the first time. It has no analogs in the available literature, except the DNA - phosphatidylethanolamine complex presented in our previous work [4]. Before the computer experiment, we presented three alternative locations of PG in the DNA double helix: either in the major (minor) groove or one of the fatty acid residues in the minor groove, but other FA residues - in the major groove. Minimizing the DNA-PG complex energy showed that in the structure with minimal energy, both linoleic acid residues are located in a minor groove (Figure 1).

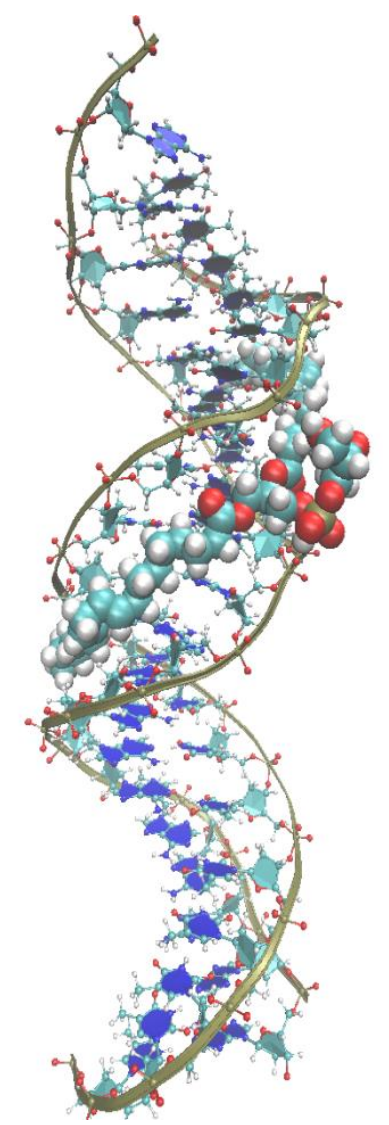

Figure 1. Molecular Dynamics of DNA Complexes with Phosphatidylglycerol. The structure of the DNA oligomer $(\mathrm{dA})_{20} \bullet(\mathrm{dT})_{20}$ with a phosphatidylglycerol molecule in the minor groove as a ligand. The optimization was carried out by molecular docking method using AutoDock in the "vina.exe" application.

Although there are no significant changes in the values of the RMSD parameter for the PG molecule in the DNA-PG complex, there are areas with symmetrical changes in the RMSD values of DNA and PG. For example, for DNA and lipid (1 Á for lipid, $1.5 \AA ̊$ for DNA), the curve decline of this parameter falls on the $1900^{\text {th }}$ step, and for lipid, the magnitude of this fall is less than $0.5 \AA$ for DNA (Figure 2). 


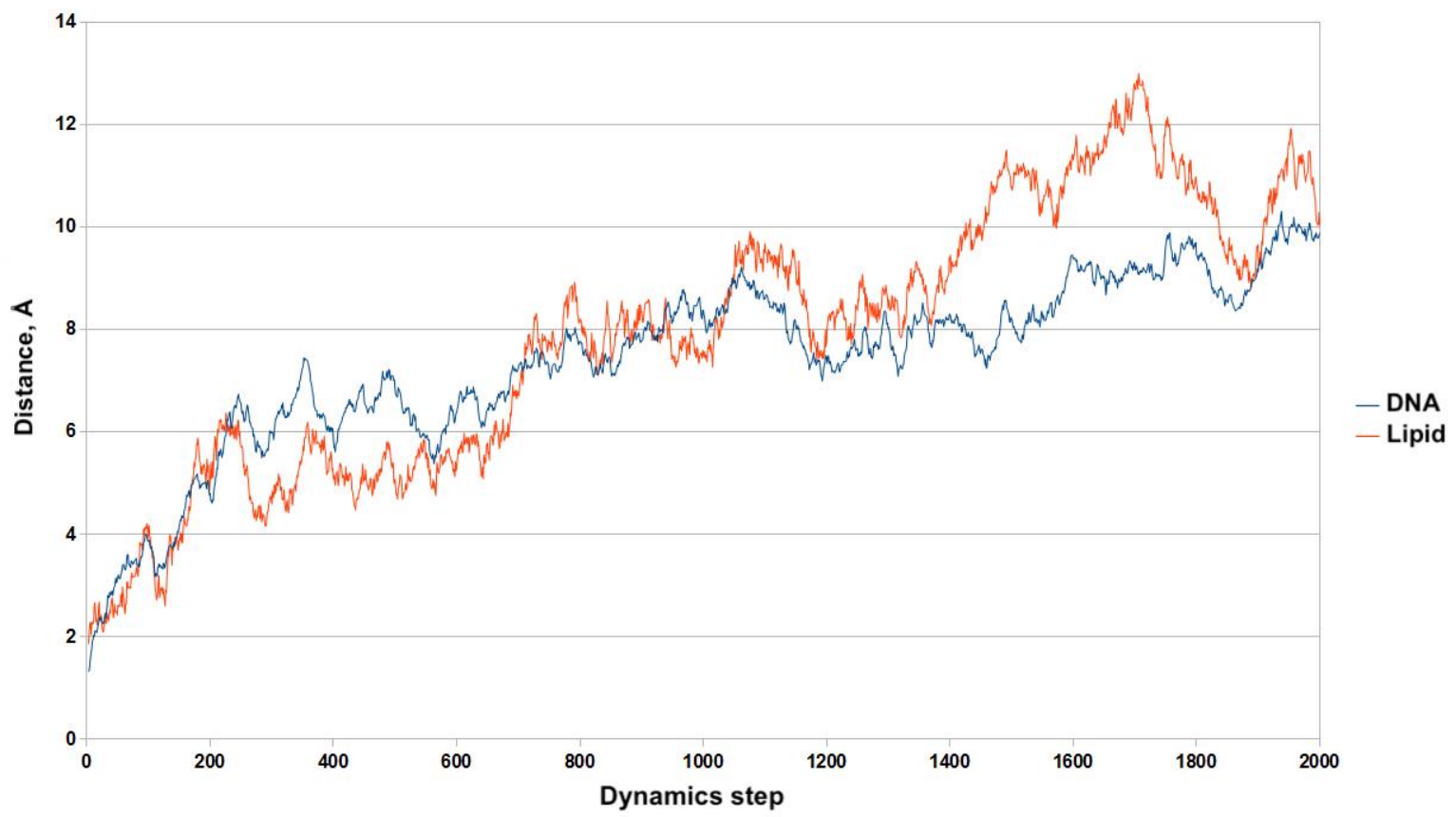

Figure 2. Molecular Dynamics of DNA Complexes with Phosphatidylglycerol. Change in "the root-meansquare deviation (RMSD)" [4] of the structures of the molecule and the ligand in the DNA complex $(\mathrm{dA})_{20} \cdot(\mathrm{dT})_{20}$ and $\mathrm{PG}$ at each saved step of the dynamics. By $\mathrm{X}$ is a step dynamics, by $\mathrm{Y}$ is a change in $\AA$ (see methods section).

The number and types of interacting atoms at the initial and final stages of the dynamics reflect the transition of structures to a more stable complex (from 14 to 254 pairs of atoms). The maximum at the intermediate steps is about 380 atom pairs. Qualitative analysis shows the increase in the number of O-H pairs. The distances between pairs of DNA and PG atoms at the initial steps of dynamics undergo a change during the entire dynamics (Figure 3). Despite this, some pairs of atoms can vary in insignificant ranges during the course of dynamics.

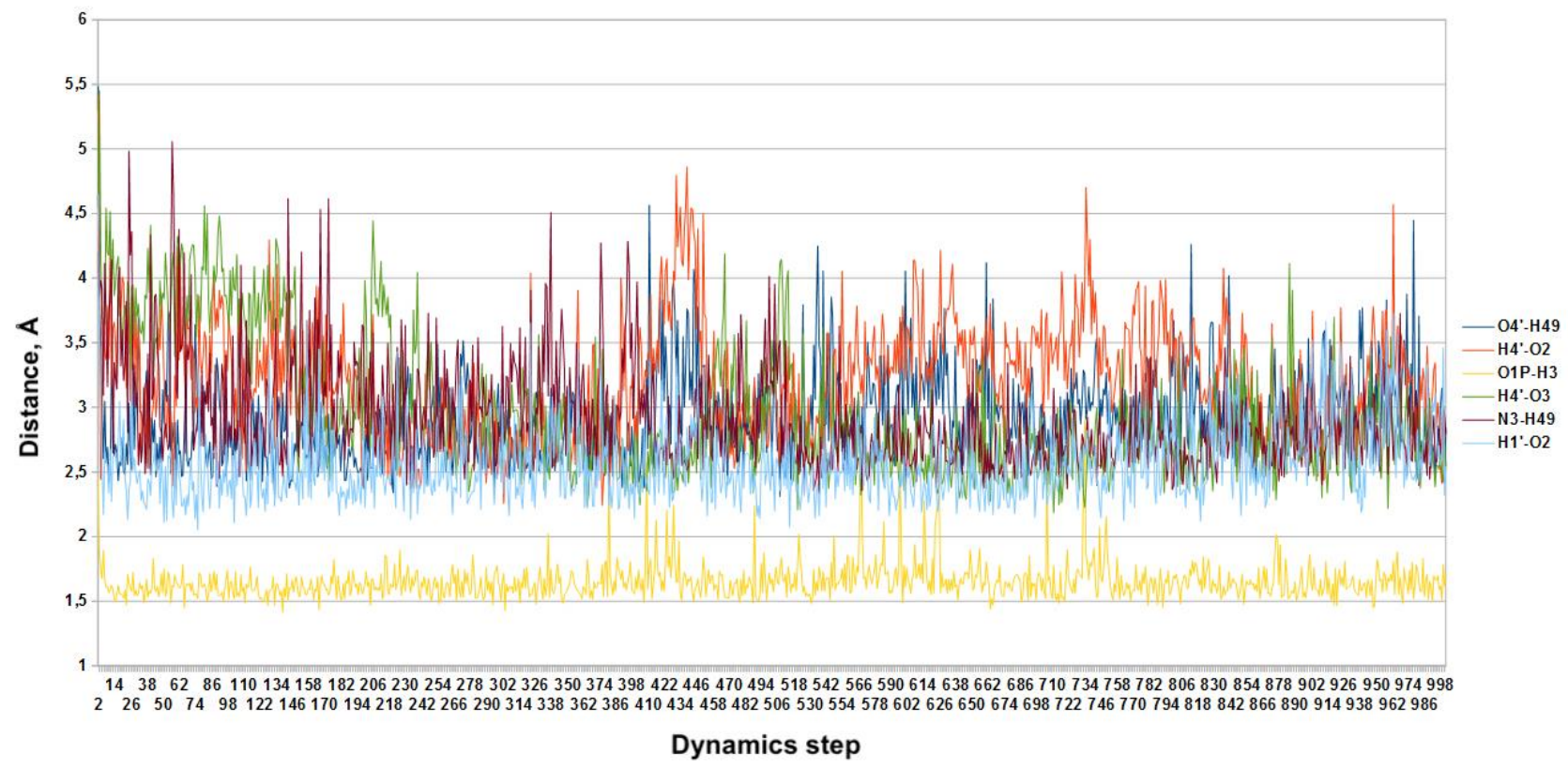

Figure 3. Molecular Dynamics of DNA Complexes with Phosphatidylglycerol. Change in the distances between pairs of atoms during dynamics, the distance between which was less than $3.4 \AA$ in the first and the last steps of dynamics. 
During the course of dynamics, the average smallest distance is observed between pairs of O1P-H3 atoms (Figure 4). The average distance value between them is $1.65 \AA$, the minimum - $1.42 \AA$ and the maximum - $2.9 \AA$. The average distance between other pairs of atoms differs by about $1 \AA$. In particular, for the pair of O4'-H49 atoms, the distance varies from 2.29 to 5.48 (the average is $2.93 \AA$ ); for the H4'-O2 pair, the distance varies from 2.24 to 5.41 (the average is $3.21 \AA$ ) ; for H4'-O3 the distance varies from 2.18 to 5.45 (the average is $2.96 \AA$ ); for N3$\mathrm{H} 49$ the distance varies from 2.33 to 5.06 (the average is $2.91 \AA$ ); for H1'-O2 - from 2.05 to 4.65 (the average is $2.56 \AA$ ).

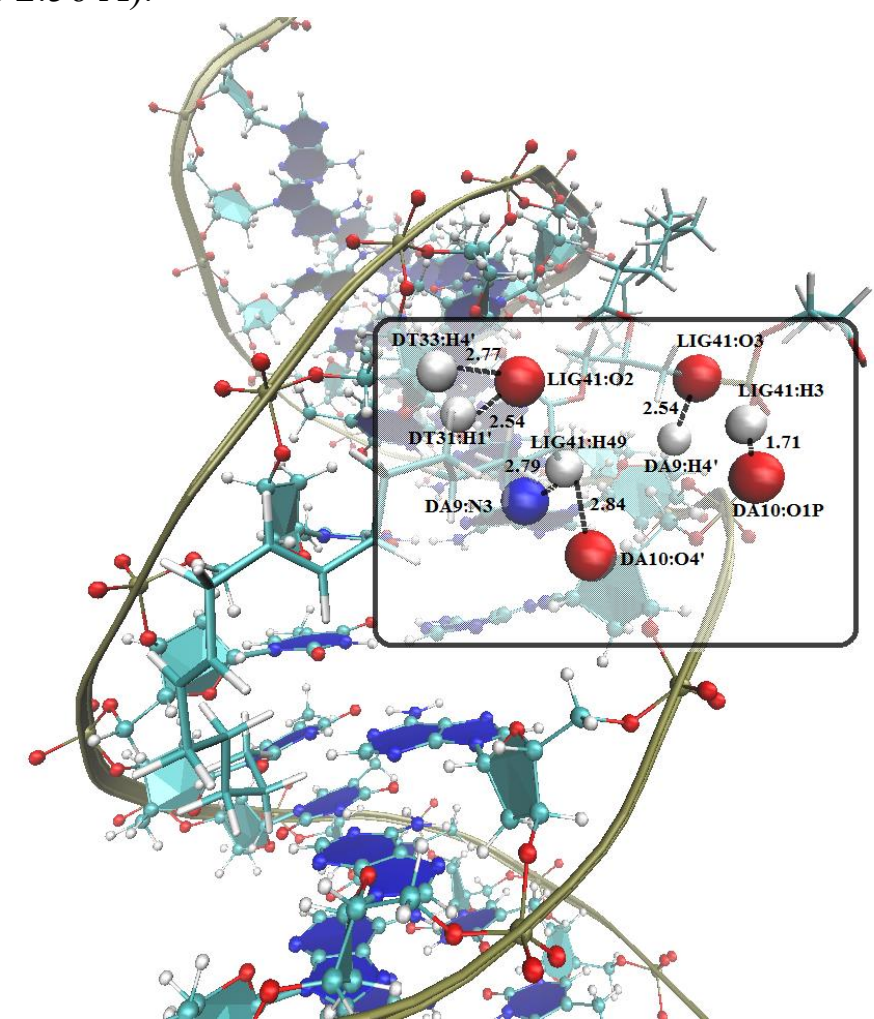

Figure 4. Molecular Dynamics of DNA Complexes with Phosphatidylglycerol. The structure of the complex of DNA oligomer and PG (neutral form). DNA chain orientation is changed to provide a better viewing angle of the complex.

The reference points for the interaction of fatty acids (neutral and anionic forms of linoleic acid) and PG with DNA are analyzed (Figure 4). The results showed that due to the presence of two fatty acids in the PG, the distance between the atoms of its fatty acids and the DNA atoms decreases by more than 2 angstroms than free fatty acids. Presumably, this is due precisely to the presence in PG of two fatty acid residues linked through the backbone of glycerophosphate. When free fatty acid interacts, the distance between the spatially similar fatty acid atoms and DNA is less than 2 angstroms. This is due to steric hindrances created by the glycerol group, i.e., close contact of the hydrophilic lipid head is complicated by glycerol, while hydrophobic tails, on the contrary, interact tightly with DNA.

\section{Conclusions}

For the first time, the interaction between a relatively long part of the DNA molecule and phosphatidylglycerol (as a ligand) along a minor groove of the DNA in aqueous solutions is shown. The structural parameters of the complex of phosphatidylglycerol with DNA are determined; the differences compared with DNA interaction with free fatty acid are explained. 
The features of the phosphatidylglycerol molecule location in the double helix of DNA are shown based on the structural peculiarities of this molecule.

\section{Funding}

This work was supported by a grant from the Russian Foundation for Basic Research, project No. 19-03-00717 (supported part 1); a grant from the Russian Science Foundation, project No. 20-16-00032 (supported part 2); the subsidy allocated to the Kazan Federal University for the fulfillment of the state assignment in the field of scientific activity (supported part 3).

\section{Acknowledgments}

The authors are acknowledging the technical support of Dr. Ilia S. Zaitsev for reference collecting and formatting.

\section{Conflicts of Interest}

The authors declare no conflict of interest.

\section{References}

1. Harvie, P.; Wong, F.M.; Bally, M.B. Characterization of lipid DNA interactions. I. Destabilization of bound lipids and DNA dissociation. Biophys J. 1998, 75, 1040-1051, https://doi.org/10.1016/S00063495(98)77593-9.

2. Wong, F.M.P.; Reimer, D.L.; Bally, M.B. Cationic lipid binding to DNA: characterization of complex formation. Biochemistry 1996, 35, 5776 -5763, https://doi.org/10.1021/bi952847r.

3. Dyachkov, P.N.; Fedorov, B.B.; Bischoff, R.; Bischoff, G; Zhdanov, R.I. DNA-phospholipid recognition: modulation by metal ion and lipid nature. Complexes structure and stability calculated by molecular mechanics. Bioelectrochemistry 2002, 58, 47-51, https://doi.org/S1567-5394(02)00132-9.

4. Zhdanov, R.I.; Kern, D.; Lorenz, W.; Ibragimova, M.Ya. Lipid and Fatty Acid Profiles of Pseudomonas aurantiaca DNA Bound Lipids Determined by Mass Spectrometry Microbiology 2015, 84, 43-49, https://doi.org/10.1134/S0026261714060228.

5. Darley, E.; Ridone, P.; Singh, J.K.D.; Wickham, S.F.J.; Baker, M.A.B. Optimised assembly of DNA-lipid nanostructures. bioRxiv 2020, 128686, https://doi.org/10.1101/2020.06.01.128686.

6. Grinde, M.T.; Skrbo, N.; Moestue, S.A.; Rodland, E.A.; Borgan, E.; Kristian, A.; Sitter, B.; Bathen, T.F.; Borresen-Dale, A.L.; Maelandsmo, G.M.; Engebraaten, O.; Sorlie, T.; Marangoni, E.; Gribbestad, I.S. Interplay of choline metabolites and genes in patient-derived breast cancer xenografts. Breast Cancer Res. 2014, 16, R5-15, https://doi.org/10.1186/bcr3597.

7. Tarasov, D.S.,; Ibragimova, M.Ya.; Izotova, E.D.; Akberova, N.I. Molecular dynamics and free energy of linoleic acid binding to DNA in an aqueous solution. Reports of the Academy of Sciences (Dokl. Biochem. Biophys.) 2012, 446, 223-228, https://doi.org/10.1134/S1607672912050043.

8. Gurr, M.I.; Harwood, J.L Lipid biochemistry. 4-th Edition. London Chapman and Hall, 1991.

9. Zaitsev S.Yu. Biological chemistry: from biologically active substances to organs and tissues of animals. Moscow ZAO "Capital Print”, 2017.

10. King, R.J.; MacBeth, M.C. Interaction of the lipid and protein components of pulmonary surfactant. Role of phosphatidylglycerol and calcium. Biochim. Biophys. Acta. 1981, 647, 159-168.

11. Chang, Y.Y.; Kennedy, E.P. Biosynthesis of phosphatidyl glycerophosphate in Escherichia coli. J. Lipid Res. 1967, 8, 447-455.

12. Zaitsev, S.Yu.; Bogolyubova, N.V; Zhang, X.; Brenig, B. Biochemical parameters, dynamic tensiometry and circulating nucleic acids for cattle blood analysis: a review. PeerJ. 2020, 8, 1-24, https://doi.org/10.7717/peerj.8997.

13. Zaitsev, S.Yu. Dynamic surface tension measurements as general approach to the analysis of animal blood plasma and serum. Advances in Colloid and Interface Science. 2016, 235, 201-213, https://doi.org/10.1016/j.cis.2016.06.007.

14. Struzik, Z.J.; Weerts, A.N.; Storch, J.; Thompson, D.H. Stereospecific synthesis of phosphatidylglycerol using a cyanoethyl phosphoramidite precursor, Chemistry and Physics of Lipids 2020, 231, 104933, https://doi.org/10.1016/j.chemphyslip.2020.104933. 
15. Watanabe, Y.; Watanabe, Y.; Watanabe, S. Structural Basis for Phosphatidylethanolamine Biosynthesis by Bacterial Phosphatidylserine Decarboxylase. Structure 2020, 28, 799-809, https://doi.org/10.1016/j.str.2020.04.006.

16. Bollag, W.B.; Joyce, N. Gonzales Phosphatidylglycerol and surfactant: A potential treatment for COVID-19? Med Hypotheses 2020, 144, 110277, https://doi.org/10.1016/j.mehy.2020.110277.

17. Luchini, A.; Vitiello, G. Mimicking the Mammalian Plasma Membrane: An Overview of Lipid Membrane Models for Biophysical Studies. Biomimetics (Basel, Switzerland) $\quad 2020,6, \quad 3$, https://doi.org/10.3390/biomimetics6010003.

18. Cattel, F.; Giordano, S.; Bertiond, C.; Lupia, T.; Corcione, S.; Scaldaferri, M.; Angelone, L.; De Rosa F.G. Use of exogenous pulmonary surfactant in acute respiratory distress syndrome (ARDS): Role in SARS-CoV2-related lung injury. Respir. Physiol. Neurobiol. 2021, 288, 103645, https://doi.org/10.1016/j.resp.2021.103645.

19. Klein, M.E.; Rieckmann, M.; Sedding, D.; Hause, G.; Meister, A.; Mäder, K.; Lucas H. Towards the Development of Long Circulating Phosphatidylserine (PS)- and Phosphatidylglycerol (PG)-Enriched AntiInflammatory Liposomes: Is PEGylation Effective? Pharmaceutics 2021, 13, 282, https://doi.org/10.3390/pharmaceutics13020282.

20. Guterres, H.; Im, W. Improving Protein-Ligand Docking Results with High-Throughput Molecular Dynamics Simulations Journal of Chemical Information and Modeling 2020, 60, 2189-2198, https://doi.org/10.1021/acs.jcim.0c00057.

21. Berenger, F.; Kumar, A.; Zhang, K.Y.J.; Yamanishi, Y. Lean-Docking: Exploiting Ligands' Predicted Docking Scores to Accelerate Molecular Docking. Journal of Chemical Information and Modeling 2021, 61, 2341-2352, https://doi.org/10.1021/acs.jcim.0c01452.

22. Winkler, D.A. Ligand Entropy Is Hard but Should Not Be Ignored. Journal of Chemical Information and Modeling 2020, 60, 4421-4423, https://doi.org/10.1021/acs.jcim.0c01146.

23. Bepari, A.K.; Reza, H.M. Identification of a novel inhibitor of SARS-CoV-2 3CL-PRO through virtual screening and molecular dynamics simulation. PeerJ 2021, 9, e11261, https://doi.org/10.7717/peerj.11261.

24. Adeleke, V.T.; Adeniyi, A.A.; Lokhat, D. Coagulation of organic pollutants by Moringa oleifera protein molecules: in silico approach. Environmental Science: Water Research \& Technology 2021, 241, e247, https://doi.org/10.1039/D1EW00247C.

25. Choudhury, C.; Bhardwaj, A. Hybrid Dynamic Pharmacophore Models as Effective Tools to Identify Novel Chemotypes for Anti-TB Inhibitor Design: A Case Study With Mtb-DapB. Frontiers in Chemistry 2020, 8, 596412, https://doi.org/10.3389/fchem.2020.596412.

26. Simon A. Bray, Tharindu Senapathi, Christopher B. Barnett, Björn A. Grüning. Intuitive, reproducible highthroughput molecular dynamics in Galaxy: a tutorial. Journal of Cheminformatics 2020, 12, 54, https://doi.org/10.1186/s13321-020-00451-6.

27. Kleywegt, G.J. Crystallographic refinement of ligand complexes. Acta Crystallogr. D. Biol. Crystallogr. 2007, 63, 94-100, https://doi.org/10.1107/S0907444906022657.

28. McCammon, J.A. Target flexibility in molecular recognition. Biochim. Biophys. Acta. 2005, 1754, 221-224, https://doi.org/10.1016/j.bbapap.2005.07.041.

29. Phillips, J.C.; Braun, R.; Wang, W.; Gumbart, J.; Tajkhorshid, E.; Villa, E.; Chipot, C.; Skeel, R.D.; Kale, L; Schulten, K. Scalable molecular dynamics with NAMD. J. Comput. Chem. 2005, 26, 1781-1802, https://doi.org/10.1002/jcc.20289.

30. Wang, J.; Wolf, R.M.; Caldwell, J.W.; Kollman, P.A.; Case, D.A. Development and testing of a general amber force field. J. Comput. Chem. 2004, 25, 1157-1174, https://doi.org/10.1002/jcc.20035.

31. Marion A, Gokcan H, Monard G. Semi-Empirical Born-Oppenheimer Molecular Dynamics (SEBOMD) within the Amber Biomolecular Package. J. Chem. Inf. Model. 2019, 59, 206-214, https://doi.org/10.1002/jcc.20035.

32. Zhang, S.; Schweitzer-Stenner, R.; Urbanc, B. Do Molecular Dynamics Force Fields Capture Conformational Dynamics of Alanine in Water? J. Chem. Theory Comput. 2020, 16, 510-527, https://doi.org/10.1021/acs.jctc.9b00588.

33. Trott, O.; Olson, A.J. AutoDock Vina: improving the speed and accuracy of docking with a new scoring function, efficient optimization, and multithreading. J. Comput. Chem. 2010, 31, 455-461, https://doi.org/10.1002/jcc.21334.

34. Coan, K.E.; Yen, T.W.F.; Carr, A.A.; Evans, D.B.; Wang, T.S. Confirmation of Parathyroid Tissue: Are Surgeons Aware of New and Novel Techniques? J. Surg. Res. 2020, 246, 139-144, https://doi.org/10.1016/j.jss.2019.08.006.

35. Hosny, N.A.; Sherif, Y. Molecular Docking Study on Some Isonicotinoyl Hydrazide Derivatives as Potential Inhibitors of COVID-19. Letters in Applied NanoBioscience 2020, 9, 1217- 1224, https://doi.org/10.33263/LIANBS93.12171224. 